\title{
Tactile Feedback for Ambient Awareness in Mobile Interactions
}

\author{
Stephen Brewster \\ Aurora Constantin \\ Glasgow Interactive Systems Group, Department of Computing Science \\ University of Glasgow, Glasgow, G12 8QQ, UK \\ stephen@dcs.gla.ac.uk
}

\begin{abstract}
The study of tactile feedback has attracted increasing interest in $\mathrm{HCl}$ over recent years. Similar to icons, tactile messages, or Tactons, can encode and transmit information through the touch sense [1]. We report an experiment to investigate if we can present contextual information to a user in a low attention, ambient manner. In this case, it is done by changing the tactile 'feel' of buttons on a touchscreen keyboard to indicate external events, for example when a friend is close by. Very short Tactons (<=300ms) on each key press were changed in roughness and rhythm to indicate the events. Results showed that users correctly identified the Tactons for the different events with a rate of 88\% when 180 Tactons were presented in 45 minutes, and $98 \%$ when the Tactons were presented in a more realistic manner. This shows that changing tactile feedback can be an effective method of presenting ambient information on a mobile device.
\end{abstract}

Tactons, tactile feedback, text entry, touchscreen mobile devices.

\section{INTRODUCTION}

Mobile devices such as smartphones have become very popular, offering a multitude of functions in one single instrument. They provide many of the features of a conventional computer but have the advantage that they can be used in a wide range of different situations and contexts. Most of these devices now have Internet connectivity and are GPS-enabled. A current research trend is directed toward using GPS information to provide services to help people locate and find friends by sharing positional information between groups, for example Google Latitude (www. google.com/ latitude), Nokia's Friend-View or Social Gravity [10]. These commonly show the locations of friends on a map so you can see who is nearby. However, if you are using other applications on the phone then it can be difficult to keep track of who is nearby. The screens of such devices are small so there is little space to display the information needed and this means the user must keep switching back to the friend finding application to check it. Our approach is to provide an ambient display of the proximity of friends so that the user does not have to switch applications. We chose to use tactile feedback as this is commonly available on mobile devices, does not disrupt visual attention that may be on the device and is less likely to be masked by environment noise than audio feedback. Previous research by Hoggan et al. [4] showed that adding simple tactile feedback for touchscreen interactions significantly improved typing performance. We extended this feedback to use different tactile cues to change the feel of the touchscreen buttons (and potentially other interactions such as clicking on links, sliding sliders, etc.) to indicate the proximity of contacts. An example scenario might be: Aurora is sitting in a café waiting for her friend Steve to turn up. She is catching up on emails using her touch-screen smartphone. As she types on the touchscreen keyboard she feels tactile feedback from the keys. As Steve gets closer she feels the buttons change as she presses them, so she knows that Steve is approaching without having to stop reading and answering her email. In this way we can present information about events such interaction. This provides a more ambient type of display than the more intrusive alerts or alarms that the phone might currently give. This paper presents a study into the possibility of using very short Tactons [1] to indicate the proximity of different contacts and to get information about the presence of an individual at a specific location. The research was based on the previous work undertaken by Hoggan et al. [4]. However, while in the previous work the purpose was to improve the touchscreen keyboard by adding tactile feedback, in the present application it was to study how very short tactile feedback can transmit information in a more ambient way under conditions of low attention.

\section{BACKGROUNDWORK}

In recent years there has been an increase in the amount of research focusing on the use of tactile feedback for interactions, especially on mobile devices. Lee et al. [6] developed a tactile et al. and Luk et al. $[9,7]$ have designed sophisticated tactile displays for hand-held computers. Hoggan et al. in 
particular, have looked at how tactile feedback for touchscreen devices can improve typing performance [4]. Touchscreens are common in mobile devices but they are smooth and featureless, la keypads. Hoggan found that by adding tactile feedback on keypresses, typing performance could be significantly improved, reducing errors and increasing speed. Results were close to those of typing on a physical keypad. She tested a range of actuators from the internal vibration motor to more sophisticated ones such as the C2 Tactor from EAI (www.eaiinfo.com) and showed that better quality actuators improved performance further. We took these results as the base for our work but used the feedback for information other than just keypresses. If we could equal the performance she achieved then we would be improving typing over a standard touchscreen while delivering more information. Several research projects have focused on the addition of tactile feedback to other types of UI widget, e.g. [5], showing that using the sense of touch can improve the usability of many types of interaction. Most research is conducted using vibrotactile actuators built in to the mobile devices; however, lateral skin stretch displays [7] and piezo-electric actuators are also becoming important research platforms. Kaaresoja et al. [5] created applications of tactile piezoelectric actuators that can provide localised feedback underneath the fingertip. The applications included text selection, scrolling and drag and drop. We wanted to see if tactile feedback could be used to provide a more ambient display of information in an interface, rather than just direct feedback on user interactions.

\subsection{Tactile Messages}

Tactons, or tactile icons, [1, 2] are structured, abstract, tactile messages which can be used to communicate information non-visually. Brown et al. [2] have studied the design of tactile messages to find the best parameters to build effective cues and the numbers of individual discriminable values within each parameter. Parameters include rhythm, roughness, body location and texture. Brown used these to create structured alerts for a mobile device based around families of different Tactons. We used Tactons as the basis for the tactile feedback in our study but rather than just giving more alerts we gave ambient feedback from other interactions with the device. The novel aspect of our Tactons is alert Tactons were up to 2.5 seconds long. However, ours had to be very short in order to keep up with the typing on a touchscreen keyboard. This reduced the number of different parameters we could use, making Tacton design for our application very challenging but extending their potential use to new areas. The rest of the paper describes the design of the Tactons we used for our study and then two experiments to test if they were effective at indicating information to users when they were typing on a touchscreen keyboard.

\section{EXPERIMENTS}

Two experiments were designed to investigate if we could create Tactons for a touchscreen keyboard that users could understand and be used to indicate proximity of friends and family when typing. We based our studies on that of Hoggan et al. [4]. A standard QWERTY touchscreen keyboard (Figure 1) was designed and implemented on a Samsung Q1 Ultra Mobile PC. It contained 56 buttons and two text areas: one to display a phrase the participants had to enter and the other one to display the text they typed (similar to that of Hoggan, see Figure 2). We used this device as it had a screen large enough for a realistically sized touchscreen keyboard along with other windows that we needed to display.

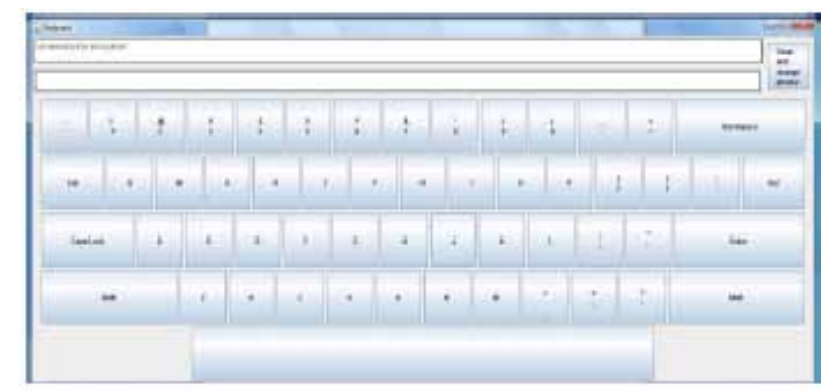

Figure 1: The touchscreen keyboard used in the experiments.

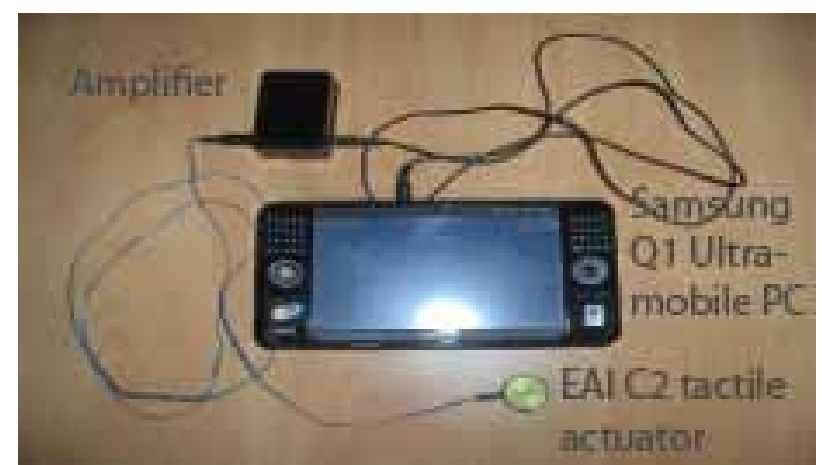

Figure 2: The experimental setup with UMPC and C2 tactor.

The C2 Tactor from EAl (www.eaiinfo.com, Figure 2 ) was used. It is a small linear vibrotactile actuator designed to provide a lightweight equivalent to large laboratory-based linear actuators. The C2 is resonant at $250 \mathrm{~Hz}$ but is also designed to produce a wide range of frequencies unlike many basic mobile phone actuators. The quality of the feedback it produces is better than a standard phone motor. It was connected to a small amplifier through the $3.5 \mathrm{~mm}$ audio jack of the UMPC and controlled via sound files. We chose this as Hoggan et al. [4] had found it to be the best actuator in their tactile keyboard study. At the start of the experiment each 
participant was asked to fix the C2 to his/her nondominant hand with an adhesive band.

\subsection{Tacton Design}

We created Tactons to represent two groups: friend and child. These reflect commonly categories suitable for testing in an experiment but could equally be used for family or any other type of information (e.g. the arrival of email from particular contacts). Each of these had three locations: far (between 100 $\mathrm{m}$ and $1 \mathrm{~km}$ ), close (within 100m) and school for child and office for the friend. Again these were arbitrary choices and could have been closer or further away (or the urgency of an email received).

Based on Brown et al. previous research into two dimensional Tactons it was decided to use roughness and rhythm for our parameters. The cues we needed had to be very short to keep pace with typing so we were unable to use complex rhythms or wave-forms. This really limited our design space to a $2 \times 3$ set. After much prototyping of different Tacotns we found it very difficult to create a set of discriminable cues larger than this (an interesting research topic would be to investigate the design of very short Tactons more deeply). The waveform (which gives the roughness of the vibration) was chosen to encode the person and rhythm to encode the location. In Brown's previous research different levels of rhythm were easier to distinguish than different levels of roughness [2].

The two waveforms were: a smooth unmodulated $250 \mathrm{~Hz}$ sine wave for child and a rough $250 \mathrm{~Hz}$ sine wave modulated by $30 \mathrm{~Hz}$ [2] for friend. Initially, the rhythms were chosen to be one, two and three notes, but after several tests it was decided to change three to four notes because the two and three note Tactons were easily confused. Both waveforms were created using Matlab and processed with Audacity. Therefore, Tactons (300ms duration) were created for the following Tacton proximity events:

T0 Child far $\begin{aligned} & \text { one-note unmodulated } 250 \mathrm{~Hz} \\ & \text { sine wave }\end{aligned}$

T1 Child close two-note unmodulated $250 \mathrm{~Hz}$ sine wave

T2 Child school four-note unmodulated $250 \mathrm{~Hz}$ sine wave

T3 Friend far one-note $250 \mathrm{~Hz}$ sine wave modulated by $30 \mathrm{~Hz}$

T4 Friend close two-note $250 \mathrm{~Hz}$ sine wave modulated by $30 \mathrm{~Hz}$
T5 Friend office

four-note $250 \mathrm{~Hz}$ sine wave modulated by $30 \mathrm{~Hz}$

A very short cue $(50 \mathrm{~ms}$ unmodulated sine wave of $250 \mathrm{~Hz}$ ) was designed to be played on pressing a key in the absence of a proximity event (Similar to Hoggan's standard keypress feedback).

\subsection{First Experiment}

We designed an initial experiment to test the effectiveness of the Tactons we had created to see if users could recognise and understand them. The experiment consisted of two phases. An initial training session allowed participants to learn the Tactons and the information encoded in them ( 2 mins), and to become familiar with the experimental procedure ( $\sim 5 \mathrm{mins})$. The interface for learning the Tactons is shown in Figure 3. This was followed by a 45 minute test in which participants were presented with 180 proximity event Tactons (30 of each type) and requested to identify them as they typed in text phrases.

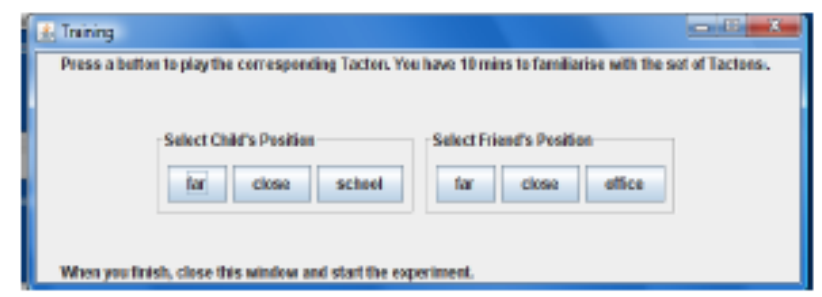

Figure 3: The Tacton training interface.

The 180 Tactons were displayed in a random order on keypresses

as participants typed. Participants were asked to identify them when they noticed a new Tacton had appeared. The events appeared while they entered a set of standard phrases (randomly selected from the MacKenzie standard 500-phrase set [8]). Each event had a random duration (about 5 s on average). That meant that during this interval of time the corresponding Tacton was played each time any of the touchscreen keys were pressed. The gap between two proximity events was $\sim 10$ s. If the Tacton was noticed and recognized by a participant he/she used the interface in Figure 4 to select the event that had occurred. This was displayed below the main keyboard window.

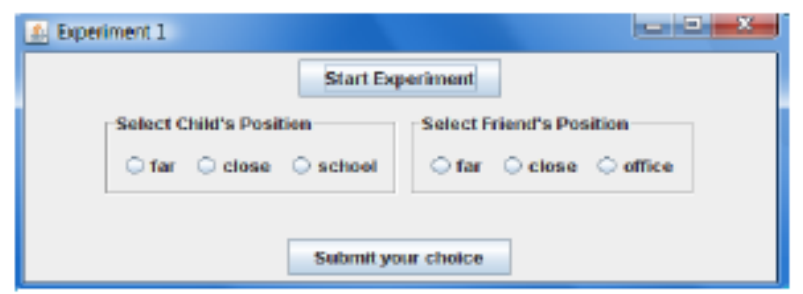

Figure 4: The Tacton response screen.

We measured the overall identification rate and the 
average time for identification of each Tacton (the time from when the user first pressed a key when the proximity event had occurred until he/she chose a button on Figure 4). In addition, the keystrokes per character (KSPC) were output to a log file. KSPC is the number of keystrokes required, on average, to generate a character of text for a given text entry technique in a given language with the ideal being one per character. It gives an indication of the accuracy of the typing as we wanted to ensure that our tactile feedback did not negatively affect typing performance. Fifteen participants took part ( 6 female and 11 male), 13 of whom were students at the University. Our main hypotheses were:

1. Participants will be able to accurately identify the Tactons;

2. Participants will be able to accurately identify individual

Tacton types with no significant differences between them;

3. The maximum confusion rate will appear between the events

encoded in Tactons with the same rhythm (same number of notes);

4. Mean response times for the identification of each type of Tacton will not be significantly different.

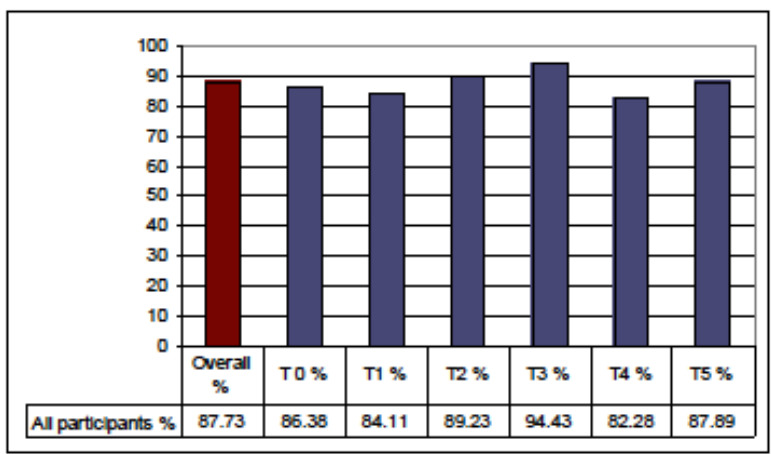

Figure 5: Overall mean Tacton recognition rates.

\subsubsection{Results from Experiment 1}

Figure 5 shows the overall recognition rates for each Tacton across all participants. The overall recognition rate was $87.7 \%$ and the rates of the different Tacton types were between $82.2 \%$ and $94.4 \%$. The overall rate is comparable with that reported by [3] for twodimensional Tactons using rhythm and location (about 87\%) and greater than that reported by Brown et al. [2] for information encoded in two-parameter Tactons (roughness and rhythm) which was $71 \%$.
However, in Hoggan et al. [3] experiment 3 rhythms and 4 location (so 12 Tactons) were used.

A one-way analysis of variance (ANOVA) was carried out on the mean recognition rates and showed no main effect $(F(2,32)=1.47, p=0.20)$. Given the very similar mean scores for each of the Tactons, the first and second hypotheses have been confirmed.

A one-way ANOVA showed no main effect for identification times for the six Tactons $(F(2,32)=0.93$, $p=0.46$ ). Again, the mean times were very similar, so hypothesis 4 can be confirmed.

Hypothesis 3 was only partially confirmed. Two unexpected results were observed by creating a confusion matrix. Maximum confusions occurred between Tacton T0 and T1, and between T0 and T4. These do not share the rhythms, which we had believed would cause the most problems. In fact T0 and T4 are the most different Tactons in our test. Further investigations are necessary to find out the cause of these results.

The results suggest that the participants could easily and accurately identify the different Tactons types indicating proximity events as they were typing on the touchscreen keyboard. This suggests that the ambient display of proximity by short tactile feedback presented on button clicks is a feasible approach.

\subsection{Second Experiment}

In the first experiment the frequency of proximity events was very high (every 10 seconds). This was done so that we could generate lots of data on Tacton recognition but was not realistic of the intended use of our system. Also, the participants got to know that a new event occurred often so could perhaps begin to predict when something was going to happen. Therefore, in the second study we reduced the rate at which proximity events occurred. This time participants had to recognize just 12 Tactons (2 of each type) in a random order, during 20 minutes. Otherwise the design of the study was identical to Experiment 1. Five participants ( 3 female and 2 male) took part, all had participated in the first experiment. This allowed us to see how users would perform as they gained more experience. Our main hypotheses were:

5. Participants will be able to recognize the Tactons more accurately than in Experiment 1;

6. Participants will be able to accurately identify individual Tacton types more accurately than in Experiment 1;

7. Average keystrokes per character (KSPC) will decrease compared to KPSC in Experiment 1.

\subsubsection{Results from Experiment 2}


The overall Tacton recognition rate was $98.3 \%$, exceeding that of the first experiment (87.73\%) and also the overall rate of identification in first experiments $(91.43 \%)$ calculated over the five people who participated in both. All the identification rates of individual Tactons increased in the second experiment (see Figure 6), except for Tacton T2 (this was due to one participant getting distracted and mistaking one of the stimuli). A one-way ANOVA showed a significant main effect $(F(4,04)=9.73$, $p=0.003$ ) between the rates of identification in the first and second experiment, confirming hypotheses 5 and 6.

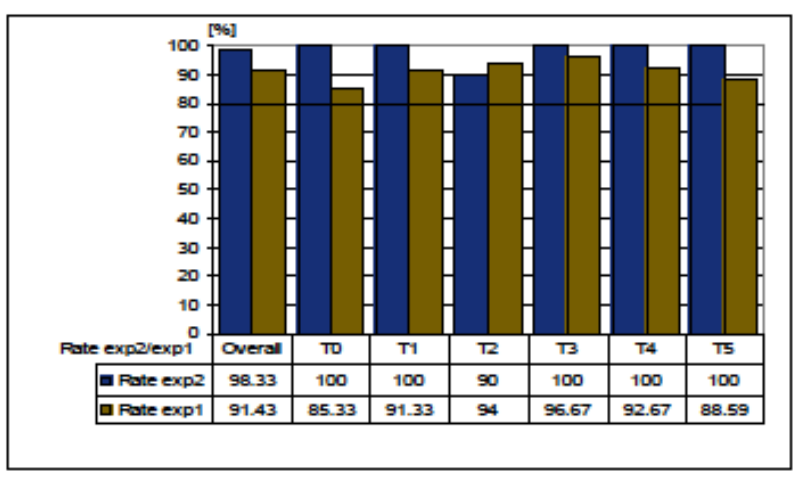

Figure 6: Comparing mean recognition rate in Expts 1 and 2.

KSPC showed a small but significant decrease from 1.0659 in Experiment 1 to 1.0063 in Experiment 2, confirming hypothesis 7 . Our KSPC rates are lower than those reported by Hoggan et al. [4], showing that the tactile feedback was helping the touchscreen typing and that people were getting better over time. The results of the second experiment show that participants were getting better at identifying the proximity events, given the more realistic setting and more practise. Accuracy rates of over $90 \%$ suggest that people could very accurately recognise different friend or family members and their proximity while performing a typing task. The reduced KSPC rates as compared to Hoggan et al. also suggest that recognising the Tactons does not come at the expense of poorer typing; participants also reduced their KSPC between the two experiments reported here.

\section{CONCLUSIONS AND FUTURE WORK}

The experimental results suggest that information can be success $\cdot$ fully transmitted in an unobtrusive way by using very short Tac•tons attached to keypress events on a touchscreen keyboard. Users were able to type accurately and still identify the proximity events quickly and correctly. The Tactons used were very short and designing them to encode the information we needed was diffi•cult; they are much shorter, for example, than those used by
Brown et al. [2] in their studies. It is interesting to see, however, that the guidelines for Tacton construction from Brown et al. still apply to these much shorter messages.

The ambient display of information via tactile feedback on mobile devices could be taken further. Other interface widgets such as buttons, scroll bars, etc. could also be given tactile feedback so that operating those would also give proximity information, while improving usability. The tactile feedback could also be used to give feedback on events other than proximity. For example, the keys could be made to feel different if an SMS or email had ar॰rived. So, if the user was browsing a Web page, he/she might feel the arrival of a message when clicking on a link. This would again give users ambient feedback about the state of their devices with•out disrupting their activity.

\section{ACKNOWLEDGMENTS}

This work was supported by the EPSRC-funded GAIME project (EP/F023405).

\section{REFERENCES}

[1] Brewster, S. and Brown, L.M. 2004. Tactons:Structured Tactile Messagesfor Non-Visual Information Display. In Proceedings of AUIC 2004. Australian Computer Society, pp 15-23.

[2] Brown, L.M., Brewster, S.A. and Purchase, H.C. A First Investigation into the Effectiveness of Tactons. In Proceed•ings of IEEE WorldHaptics 2005. IEEE Press, pp 167-176.

[3] Hoggan, E., Anwar, S. and Brewster, S.A. Mobile Multi-Actuator Tactile Displays. In Proceedings of 2nd Interna tional Workshop Haptic and Audio Interaction Design 2007. Springer LNCS, pp 22-33.

[4] Hoggan, E, Brewster, S.A. and Johnston, J. Investigating the Effectiveness of Tactile Feedback for Mobile Touchscreens.

In Proceedings of ACM CHI2008. ACM Press, pp 1573-1582.

[5] Kaaresoja, T., Brown, L. M. and Linjama, J. Snap-Crackle Pop: Tactile Feedback for Mobile TouchScreens. In Pro ceedings of Eurohaptics 2006, pp $565-566$.

[6] Lee, J.C., Dietz, P., Leigh, D., Yerazunis, W. and Hudson,

S.E. Haptic Pen: ATactile Feedback Stylusfor Touch Screens. In Proceedings of ACM UIST 2004, ACM Press, pp 291-294.

[7] Luk, J., Pasquero, J., Little, S., MacLean, K., Levesque, V. and Hayward, V. A role for haptics in mobile interaction. In Proceedings of $\mathrm{ACM} C \mathrm{CH}$ 2006, ACM Press, 171-180.

[8] Mackenzie, I.S., Soukoreff, R., W. 2003: 
Phrase sets for evaluating text entry techniques. In Extended Abstracts of ACM CHI 2003, ACM Press, pp. 754-755.

[9] Poupyrev, I. and Maruyama, S., Tactile Interfacesfor Small TouchScreens. In Proceedings of ACM UIST 2003, ACM Press, 217-220.

[10] Williamson, J., Robinson, S., Stewart, S., Murray-Smith, R. and Brewster, S. Social Gravity: AVirtual Elastic Tether for Casual, Privacy-Preserving Pedestrian Rendezvous. In Pro•ceedings of ACM CHI 2010. ACM Press, pp 1485-1494. 